\title{
A six-month double-blind, placebo-controlled, randomized clinical trial of duloxetine for the treatment of fibromyalgia
}

\author{
Amy S Chappell' \\ Laurence A Bradley ${ }^{2}$ \\ Curtis Wiltse' \\ Michael J Detke 1,3,4 \\ Deborah N D'Souza' \\ Michael Spaeth ${ }^{5}$ \\ 'Lilly Research Laboratories, \\ Indianapolis, IN, USA; ${ }^{2}$ University \\ of Alabama at Birmingham, \\ Birmingham, Alabama, USA; ${ }^{3}$ ndiana \\ University School of Medicine, \\ Indianapolis, IN, USA; ${ }^{4}$ Harvard \\ Medical School, Boston, MA, USA; \\ ${ }^{5}$ Practice for Internal Medicine/ \\ Rheumatology, Graefelfing, Germany
}

Correspondence: Amy S Chappell Eli Lilly and Company, Lilly Corporate Center, Drop Code 6I I2, Indianapolis, IN 46285, USA

Tel +I 3172773846

Fax +I 3172766026

Email aschappell@lilly.com
Objective: Assess the efficacy of duloxetine $60 / 120 \mathrm{mg}(\mathrm{N}=162)$ once daily compared with placebo $(\mathrm{N}=168)$ in the treatment of patients with fibromyalgia, during six months of treatment.

Methods: This was a phase-III, randomized, double-blind, placebo-controlled, parallel-group study assessing the efficacy and safety of duloxetine.

Results: There were no significant differences between treatment groups on the co-primary efficacy outcome measures, change in the Brief Pain Inventory (BPI) average pain severity from baseline to endpoint $(P=0.053)$ and the Patient's Global Impressions of Improvement (PGI-I) at endpoint $(P=0.073)$. Duloxetine-treated patients improved significantly more than placebo-treated patients on the Fibromyalgia Impact Questionnaire pain score, BPI least pain score and average interference score, Clinical Global Impressions of Severity scale, area under the curve of pain relief, Multidimensional Fatigue Inventory mental fatigue dimension, Beck Depression Inventory-II total score, and 36-item Short Form Health Survey mental component summary and mental health score. Nausea was the most common treatment-emergent adverse event in the duloxetine group. Overall discontinuation rates were similar between groups.

Conclusions: Although duloxetine $60 / 120 \mathrm{mg}$ /day failed to demonstrate significant improvement over placebo on the co-primary outcome measures, in this supportive study, duloxetine demonstrated significant improvement compared with placebo on numerous secondary measures.

Keywords: fibromyalgia, duloxetine, placebo, double-blind, trial, pain

\section{Introduction}

Fibromyalgia is a chronic pain disorder characterized by widespread pain, tenderness, fatigue, sleep difficulties, and stiffness. ${ }^{1-3}$ Fibromyalgia occurs in about $2 \%$ of the general population of the United States and is more prevalent in women (estimated at $3.4 \%$ to $10.5 \%)$ than in men $(0.5 \%) .4,5$

Serotonin and norepinephrine have been implicated in the mediation of endogenous analgesic mechanisms via the descending inhibitory pain pathways in the brain and spinal cord. ${ }^{6,7}$ An imbalance in these inhibitory mechanisms may contribute to central sensitization and hyperexcitability of the spinal and supraspinal pain-transmitting pathways. This imbalance may manifest as persistent pain. ${ }^{8}$ Dysfunction in both serotonin and norepinephrine systems has been implicated in the etiology of fibromyalgia. ${ }^{9-12}$ Drugs often administered to treat fibromyalgia include analgesics, ${ }^{13}$ nonsteroidal antiinflammatory agents, ${ }^{14}$ sedatives, ${ }^{15}$ antidepressants, ${ }^{16}$ and selective serotonin reuptake inhibitors. ${ }^{17}$ In a 12-week trial, the serotonin and norepinephrine reuptake inhibitor milnacipran was shown to relieve fibromyalgia symptoms at a dose of $200 \mathrm{mg} .{ }^{18}$

Duloxetine hydrochloride $\left(\mathrm{Cymbalta}^{\circledR}\right)$ is a selective serotonin and norepinephrine reuptake inhibitor that is relatively balanced in its affinity for both serotonin and 
norepinephrine reuptake inhibition. Preclinical models of central sensitization suggest that duloxetine will have efficacy in the treatment of persistent/chronic pain. In rodents, duloxetine has demonstrated efficacy in the formalin and capsaicin models of persistent pain, as well as in the partial sciatic nerve ligation ${ }^{19}$ and L5/L6 spinal nerve ligation models of neuropathic pain. ${ }^{20}$ Patients with fibromyalgia have quantitatively altered nociception compared with pain-free patients, suggesting that people with fibromyalgia process sensory information differently, most likely due to changes in the central processing of pain at the spinal level. ${ }^{21}$ Current knowledge of fibromyalgia as a persistent pain condition due to central sensitization, along with the efficacy of duloxetine in preclinical analgesic models of central sensitization, suggests that duloxetine will have efficacy in the treatment of fibromyalgia.

Duloxetine is efficacious in the treatment of the painful physical symptoms associated with depression ${ }^{22}$ and the pain associated with diabetic neuropathy in nondepressed patients. ${ }^{23,24}$ Two double-blind, placebo-controlled, 12-week clinical trials of duloxetine have demonstrated its efficacy in patients with fibromyalgia with and without major depressive disorder (MDD). The first study ${ }^{25}$ examined the safety and efficacy of duloxetine $60 \mathrm{mg}$ twice daily (BID) in male and female patients and the second study ${ }^{26}$ evaluated duloxetine $60 \mathrm{mg}$ once daily (QD) and $60 \mathrm{mg}$ BID in female patients. ${ }^{26}$ The first study showed a significant effect on reduction of pain in women but not in men. The small number of men in the study $(\mathrm{N}=25)$ may explain the unexpected discrepancy between results in men and women.

On the basis of the evidence that duloxetine (60 mg QD and/or $60 \mathrm{mg}$ BID) was safe and efficacious in the treatment of fibromyalgia for 12 weeks, ${ }^{25,26}$ the following trial was conducted to assess the efficacy in pain reduction, as well as safety, of duloxetine 60/120 mg QD compared with placebo during six months of therapy.

\section{Methods}

\section{Overview}

This multicenter study (F1J-MC-HMEF) was conducted in 36 study centers in Germany, Spain, Sweden, the United Kingdom, and the United States. The study settings included outpatients from private practices and university clinics. Enrollment began in September 2005, and the study was completed in December 2006. Each site's Institutional Review Board approved the protocol, and all patients provided written informed consent before study procedures were initiated.

\section{Entry criteria}

Patients were eligible for the study if they were $\geq 18$ years of age and met criteria for fibromyalgia as defined by the American College of Rheumatology (ACR), ${ }^{1}$ with or without MDD. Exclusion criteria included: current or previous treatment with duloxetine; any current primary Axis I diagnosis other than MDD (defined by the Diagnostic and Statistical Manual of Mental Disorders, Fourth Edition); pain symptoms related to traumatic injury, structural rheumatic disease, or regional rheumatic disease (such as osteoarthritis, bursitis, tendonitis); regional pain syndrome; multiple surgeries or failed back syndrome; confirmed current or previous diagnosis of rheumatoid arthritis, inflammatory arthritis, infectious arthritis, or an autoimmune disease; and serious medical illness.

\section{Study design}

This was a phase-III, parallel, double-blind, placebo-controlled study of patients who met the ACR criteria for fibromyalgia. ${ }^{1}$ The study design is illustrated in Figure 1. Patients were randomly assigned to either duloxetine $60 \mathrm{mg}$ QD or placebo in a 1:1 ratio. Assignment to treatment groups was determined by a computer-generated random sequence within each study center, stratified by MDD status (yes, no).

Following the one-week screening phase, patients were treated in a double-blind manner for 27 weeks. Patients randomly assigned to the duloxetine $60 \mathrm{mg}$ QD treatment group underwent a titration in which they received duloxetine $30 \mathrm{mg}$ QD for one week before receiving duloxetine $60 \mathrm{mg}$ QD for 12 weeks. If at Visit 8 (Week 13) the patient did not have $\geq 50 \%$ reduction in the Brief Pain Inventory-Modified Short Form (BPI) ${ }^{27}$ average pain score, the patient was blindly escalated to $120 \mathrm{mg}$ QD. If the patient could not tolerate this dose, the patient was allowed to return to the 60 -mg dose. Patients were allowed to increase their dose to $120 \mathrm{mg}$ any time between Visits 8 and 10 (Weeks 13 and 23), based upon whether they had $\geq 50 \%$ reduction in their BPI average pain score. If at any time between Visits 9 and 11 (Weeks 18 and 27) the patient had tolerability issues with the higher dose (120 mg QD), the patient was allowed to go back to the lower dose (60 mg QD).

\section{Outcome measures}

\section{Co-primary efficacy outcome measures}

Reduction of pain severity was measured by the change in the average pain item of the $\mathrm{BPI}^{27}$ from baseline to endpoint; patient-reported improvement was assessed by the Patient's Global Impressions of Improvement (PGI-I) questionnaire ${ }^{28}$ at endpoint. 


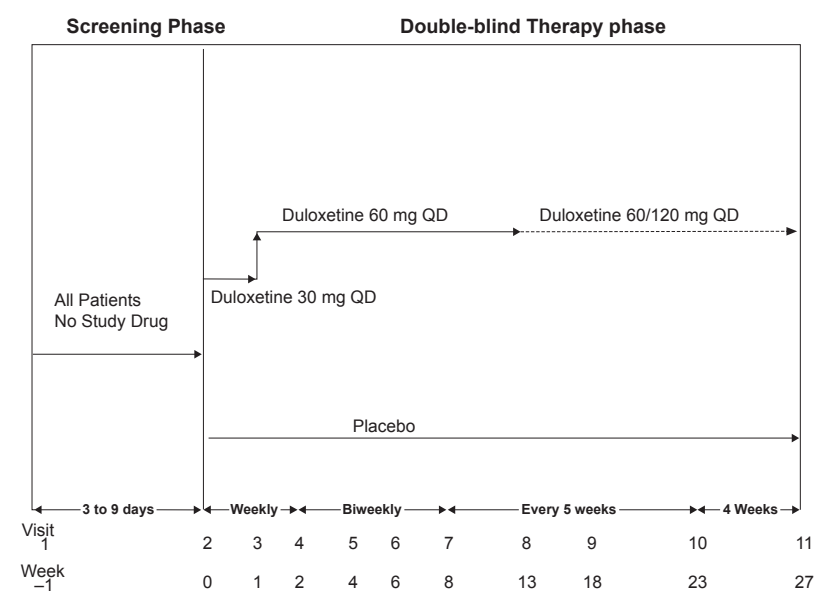

Figure I Study design.

\section{Secondary efficacy measures}

The Fibromyalgia Impact Questionnaire (FIQ ${ }^{29}$ is a selfadministered questionnaire that measures fibromyalgia patient status, progress, and outcomes over the past week. The total score ranges from 0 to 80 . A higher score indicates a more negative impact. The Clinical Global Impressions of Severity (CGI-Severity) ${ }^{28}$ scale evaluated the severity of illness at the time of assessment. The score ranges from 1 (normal, not at all ill) to 7 (among the most extremely ill patients). The CGI-Severity is administered by a study physician (blinded to study treatment) in the presence of the patient. The tender point pain threshold ${ }^{30}$ was assessed using a dolorimeter for all 18 tender points by a study physician or qualified study personnel (blinded to study treatment), and the mean of the thresholds and the number of tender points with a low threshold $\left(\leq 4 \mathrm{~kg} / \mathrm{cm}^{2}\right)$ were evaluated. The area under the curve (AUC) of pain relief was derived from the BPI average pain score. ${ }^{27}$ The BPI severity score (worst pain, least pain, average pain in the past 24 hours, pain right now) and average interference pain score are self-reported subscales that measure the severity of pain and the interference of pain on function. The severity scores range from 0 (no pain) to 10 (pain as bad as you can imagine). The interference scores range from 0 (does not interfere) to 10 (completely interferes) and include seven questions assessing the interference of pain in the past 24 hours for general activity, mood, walking ability, normal work, relations with other people, sleep, and enjoyment of life. The Multidimensional Fatigue Inventory $(\mathrm{MFI})^{31}$ is a 20 -item, self-report instrument designed to collect data on the following dimensions: general fatigue, physical fatigue, mental fatigue, reduced motivation, and reduced activity. Each dimension is calculated as the sum of four statements regarding fatigue on a 0 to 5 scale, and thus, the score ranges between 0 and 20, with a higher score indicating a higher degree of fatigue. The 17-item Hamilton Depression Rating Scale $\left(\mathrm{HAMD}_{17}\right)^{32,33}$ is a widely used observational rating measure of depression severity and its improvement during the course of therapy. The HAMD total score ranges from 0 (not at all depressed) to 52 (severely depressed). The Beck Depression Inventory-II (BDI-II) ${ }^{34}$ is a 21-item patient-completed questionnaire designed to assess characteristics of depression. Each item is rated on a four-point scale ( $0=$ not present; $3=$ present in the extreme). The total score ranges from 0 to 63 ; the higher the score, the more severe the depressive symptoms.

\section{Health outcome and quality of life measures}

These included the patient-rated Sheehan Disability Scale (SDS), ${ }^{35}$ the 36-item Short Form Health Survey (SF-36) ${ }^{36}$ and the European Quality-of-Life Questionnaire - 5 Dimensions (EQ-5D). ${ }^{37}$

\section{Safety}

The safety and tolerability of duloxetine were assessed by evaluation of spontaneously reported adverse events, reasons for discontinuation, laboratory tests (hematology, clinical chemistry, lipids), vital signs, weight, and electrocardiograms (ECG).

\section{Statistical analysis}

This study required the enrollment of 320 patients to have at least $80 \%$ power to detect a treatment group difference of -1.2 points in the baseline-to-endpoint mean change in BPI average pain score between duloxetine 60/120 mg QD and placebo, assuming a common standard deviation (SD) of 2.90, and the treatment group difference of 0.69 with a common SD of 1.68 for the endpoint PGI-I score. In the sample size calculation, a two-sided test with a significance level of 0.05 and a discontinuation rate of $40 \%$ were used.

All analyses were conducted on an intent-to-treat (ITT) basis. An ITT analysis is an analysis of data by the groups to which patients were assigned by random allocation, even if the patient did not take the assigned treatment, did not receive the correct treatment, or otherwise did not follow the protocol. All analyses, except response at endpoint based on $\geq 30 \%$ reduction in the BPI average pain score, were defined a priori (before unblinding) in the protocol or statistical analysis plan. Treatment effects were evaluated based on a two-sided significance level of 0.05 and interaction effects at a significance level of 0.10 . No adjustments 
for multiple comparisons were made. Unless otherwise specified, when a total score was calculated from individual items, it was considered missing if any of the individual items were missing. For the SDS item of "work," if a patient had not worked/studied at all during the past week for reasons unrelated to the disorder, this item was imputed by using the mean score from the other two items of the SDS for that patient. When a mean score was computed from individual items, it was calculated from existing values.

An analysis-of-covariance (ANCOVA) model was the primary analytic method used to analyze continuous efficacy variables, overall and within subgroups, where the model contained the main effects of treatment and investigator, with the baseline score as a covariate (Patient's Global Impressions of Severity [PGI-S] for the analysis of the PGI-I). The treatment-by-investigator interaction was tested using a separate ANCOVA. When the interaction was statistically significant, the nature of the interaction was explored, but the treatment effect was evaluated using the model without the interaction term. The consistency of the effect of duloxetine compared with placebo on the BPI average pain score in patient subgroups of age $(<65, \geq 65)$, sex, race (Caucasian, other), major depressive disorder (yes, no), secondary diagnosis of anxiety (yes, no), and previous antidepressant use (yes, no) was investigated by adding the relevant subgroup and treatment-by-subgroup interaction terms to the ANCOVA model. Continuous demographic and baseline data, vital signs, ECG intervals and heart rate, and ranked laboratory data were analyzed using an analysis-of-variance (ANOVA) model that contained the main effects of treatment and investigator. Type II sums-of-squares for the least-squares (LS) mean were used for the statistical comparison using ANOVA or ANCOVA. Categorical variables were compared between treatment groups using Fisher's exact test.

Some efficacy variables measured repeatedly over time were analyzed using a likelihood-based mixed-effects model repeated measures (MMRM) approach, ${ }^{38}$ to better understand the time profile of response. The MMRM analyses use likelihood-based estimation, and subject-specific effects. Correlations between repeated measures are developed through the within-subject error correlation structure. The model included the fixed categorical effects of treatment, investigator, visit, and treatment-by-visit interaction, as well as the continuous fixed covariates of baseline score and baseline score-by-visit interaction. Type III sums-of-squares for the LS mean were used.

Treatment-emergent adverse events (TEAEs) were defined as events that first occurred or worsened after randomization as compared with the maximum prerandomization severity. Events were reported using preferred terms for Version 9.1 of MedDRA ${ }^{\circledR}$ (Medical Dictionary for Regulatory Activities) terminology. ${ }^{39}$

Unless otherwise specified, "baseline" refers to the last nonmissing observation at or before the randomization visit (Visit 2, Week 0), and "endpoint" refers to the last nonmissing observation in the time period of analysis. The baseline used for determination of elevated blood pressure (BP) was the maximum prerandomization observation. When the investigator sites were used in the analyses, the sites having fewer than eight randomly assigned patients with a nonmissing value for baseline-to-endpoint change in the BPI average pain score were pooled and considered a single site. If a pooled site still had fewer than eight randomly assigned patients, these patients were pooled with the smallest remaining site. This pooling procedure continued until every site used in the analyses had at least eight patients with data for change in the BPI average pain score.

All statistical analyses were performed using $\mathrm{SAS}^{\circledR}$, Version 9.1.3 (SAS Institute Inc., Cary, NC, USA), running on a UNIX ${ }^{\circledR}$ system using SAS ${ }^{\circledR}$ Drug Development. Throughout this article, the term "significant" indicates statistical significance, and "mean" refers to LS mean, except for demographic and clinical characteristics at baseline and for laboratory tests, for which it refers to the arithmetic mean.

\section{Results}

\section{Patient disposition}

A total of 330 patients who met the entry criteria were randomly assigned to duloxetine $60 \mathrm{mg}$ QD $(\mathrm{N}=162)$ or placebo $(\mathrm{N}=168)$ (Figure 2). A significant difference was seen in rates of patients discontinuing due to lack of efficacy (duloxetine, 12 [7.4\%], placebo 25 [14.9\%], $P=0.036$ ).

\section{Baseline demographic and clinical characteristics}

The majority of the patients were female (93.3\%) and Caucasian (90.9\%), followed by Hispanic (7.6\%), and African descent $(0.9 \%)$. The mean (SD) age of the enrolled patients was 50.5 (10.7) years, and $22.4 \%$ of the enrolled patients had current MDD. No significant differences among treatment groups were observed in any of the patient demographics or clinical characteristics (Table 1).

\section{Efficacy}

Although the co-primary efficacy measures of mean change in the BPI average pain score from baseline to endpoint 


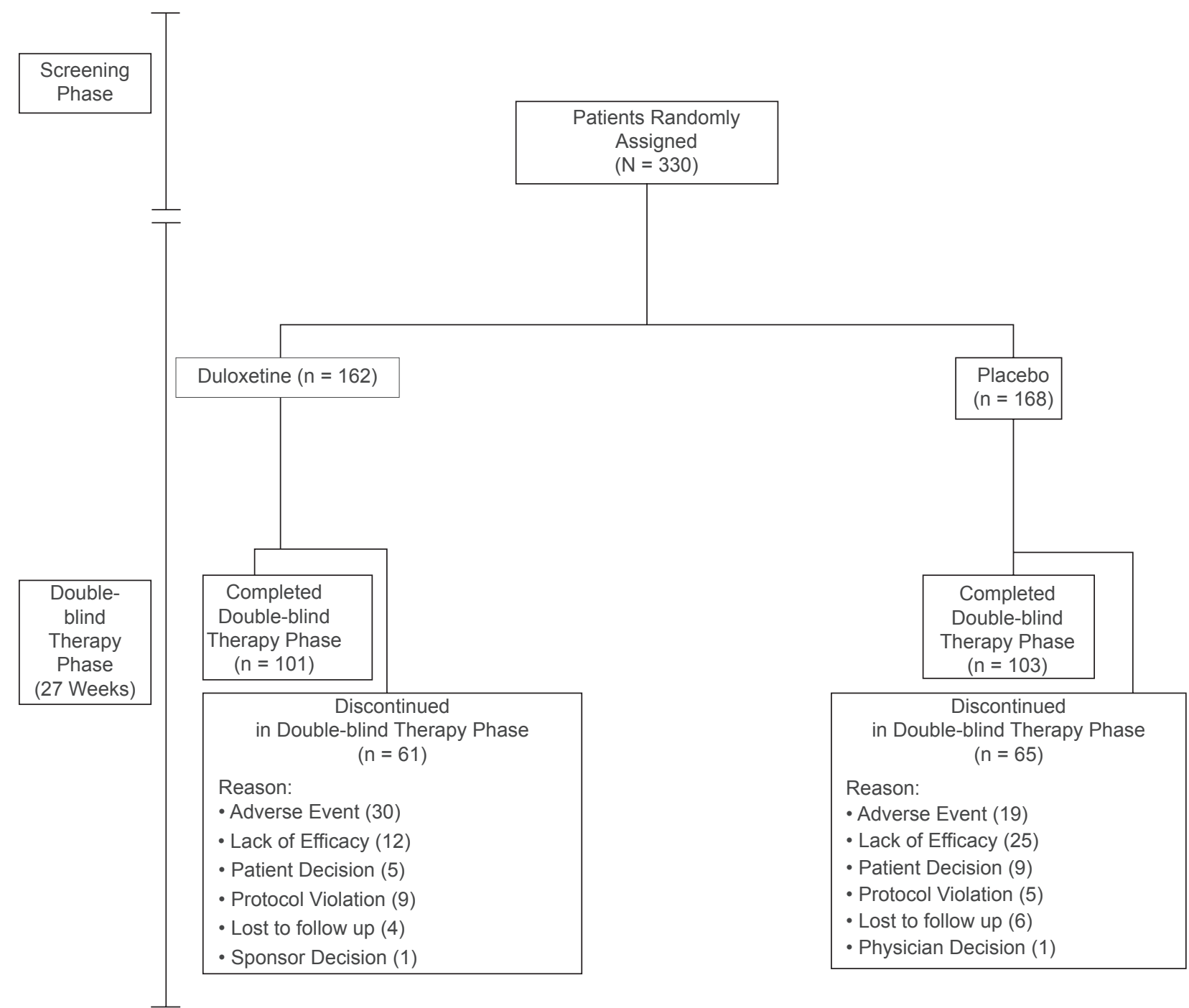

Figure 2 Patient disposition.

and the mean PGI-I at endpoint showed greater numerical improvement for duloxetine- than placebo-treated patients, the differences between the treatment groups were not statistically significant (Table 2). For these, and several other variables, a significant treatment-by-investigator interaction was observed (Table 2). The nature of the interaction was investigated, and it could not be clearly explained by country-specific or geographic region (United States vs Europe) effects. The duloxetine group improved significantly more than the placebo group at all visits through Week 8 on both co-primary measures (BPI average pain score: Week 1 $P<0.001$, Week $2 P<0.001$, Week $4 P=0.001$, Week 6 $P=0.002$, Week $8 P=0.015$; PGI-I: Week $1 P=0.043$, Week $2 P=0.005$, Week $4 P=0.014$, Week $6 P=0.003$, Week $8 P=0.007)$ as well as at Week $18(P=0.014)$ for the BPI average pain score, and Weeks $18(P=0.008)$,
$23(P=0.002)$, and $27(P=0.003)$ for the PGI-I, from the repeated measures analyses. Duloxetine-treated patients had significantly greater AUC of pain relief (mean AUC $=270.07$ ) than placebo-treated patients (mean $\mathrm{AUC}=185.25 ; P=0.010$ ) (Table 2). Compared with the placebo group, the duloxetine group had a significantly greater improvement from baseline to endpoint in the BPI least pain score (mean change: duloxetine -1.22 , placebo $-0.73, P=0.046$ ) and average interference score (mean change: duloxetine -1.69 , placebo -1.03 , $P=0.009$ ). In addition, significant between-group differences were observed in the FIQ pain score (mean change: duloxetine -1.69 , placebo $-1.06, P=0.030$ ), CGI-S (mean change: duloxetine -0.57 , placebo $-0.28, P=0.011$ ), MFI mental fatigue score (mean change: duloxetine -0.99 , placebo $-0.03, P=0.023$ ), and BDI-II total score (mean change: duloxetine -3.42 , placebo $-1.45, P=0.017$ ) (Table 2). 
Table I Demographic and clinical characteristics at baseline

\begin{tabular}{|c|c|c|c|}
\hline Variable & $\begin{array}{l}\text { Duloxetine 60/1 } 20 \mathrm{mg} \text { QD } \\
N=162\end{array}$ & Placebo $N=168$ & $P$ value \\
\hline Age (years), mean (SD) & $50.75(10.05)$ & $50.23(11.35)$ & 0.723 \\
\hline \multicolumn{4}{|l|}{ Sex } \\
\hline Female, n (\%) & $149(91.98)$ & $159(94.64)$ & \multirow[t]{2}{*}{0.382} \\
\hline Male, n (\%) & $13(8.02)$ & $9(5.36)$ & \\
\hline \multicolumn{4}{|l|}{ Race (origin) } \\
\hline Caucasian, n (\%) & 150 (92.59) & $150(89.29)$ & \multirow[t]{5}{*}{0.555} \\
\hline African, n (\%) & $2(1.23)$ & I $(0.60)$ & \\
\hline Hispanic, n (\%) & $10(6.17)$ & $15(8.93)$ & \\
\hline Native American, n (\%) & 0 & I $(0.60)$ & \\
\hline West Asian, n (\%) & 0 & I $(0.60)$ & \\
\hline Weight (kg), mean (SD) & $77.7 \mid(16.79)$ & $77.03(19.24)$ & 0.739 \\
\hline \multicolumn{4}{|l|}{ Diagnosis of MDD } \\
\hline No, n (\%) & $126(77.78)$ & I 30 (77.38) & \multirow[t]{2}{*}{1.00} \\
\hline Yes, n (\%) & $36(22.22)$ & $38(22.62)$ & \\
\hline \multicolumn{4}{|l|}{ Secondary diagnosis of anxiety } \\
\hline No, n (\%) & $152(98.06)$ & $165(98.80)$ & \multirow[t]{2}{*}{0.675} \\
\hline Yes, n (\%) & $3(1.94)$ & $2(1.20)$ & \\
\hline \multicolumn{4}{|l|}{ Previous antidepressant use } \\
\hline No, n (\%) & $91(56.17)$ & $96(57.14)$ & \multirow[t]{2}{*}{0.912} \\
\hline Yes, n (\%) & $71(43.83)$ & $72(42.86)$ & \\
\hline BPI average pain score, mean (SD) & $6.58(1.52)$ & $6.43(1.48)$ & 0.380 \\
\hline FIQ total score, mean (SD) & $49.55(11.28)$ & $50.62(12.47)$ & 0.380 \\
\hline Mean tender point threshold, mean (SD) & $2.46(0.92)$ & $2.31(0.74)$ & 0.092 \\
\hline Count of low threshold, mean (SD) & $16.38(2.90)$ & $16.81(2.10)$ & 0.128 \\
\hline CGI-S, mean (SD) & $3.81(1.20)$ & $3.79(1.26)$ & 0.747 \\
\hline PGI-S, mean (SD) & $3.97(1.32)$ & $3.84(1.36)$ & 0.388 \\
\hline $\mathrm{HAMD}_{17}$ total score, mean (SD) & $9.32(5.27)$ & $9.31(5.4 I)$ & 0.865 \\
\hline Non-MDD patients, mean (SD) & $7.98(4.27)$ & $7.8 I(4.48)$ & 0.621 \\
\hline MDD patients, mean (SD) & $13.92(5.85)$ & $14.39(5.24)$ & 0.625 \\
\hline
\end{tabular}

Notes: Some percentages do not add up to 100 due to rounding. For some variables, not all patients had data.

Abbreviations: QD, once daily; SD, standard deviation; MDD, major depressive disorder; BPI, Brief Pain Inventory; FIQ, Fibromyalgia Impact Questionnaire; CGI-S, Clinical Global Impressions of Severity; PGI-S, Patient's Global Impressions of Severity; HAMD ${ }_{17}$, 7 -item Hamilton Depression Rating Scale.

Analysis of the BPI average pain severity score response rates (defined as $\geq 50 \%$ reduction from baseline to endpoint) revealed no significant differences for the duloxetine group (29.1\% [46/158], $P=0.455)$ compared with the placebo group $(25.1 \%$ [42/167]). Analysis of the response rates at endpoint, based on a $\geq 30 \%$ reduction in the BPI average pain severity score, also revealed no significant difference between the duloxetine (38.0\% [60/158]; $P=0.355)$ and placebo groups (32.9\% [55/167]). Sustained response was defined as $\geq 50 \%$ reduction from baseline to endpoint in the BPI average pain severity, with $\mathrm{a} \geq 50 \%$ reduction from baseline at an earlier visit, and with at least a $30 \%$ reduction from baseline at every visit with data in between. Analysis of the BPI average pain severity score sustained response showed no significant difference for the duloxetine group (23.4\% [37/158], $P=0.272$ ) compared with the placebo group (18.0\% [30/167]).

For therapy-by-subgroup evaluation of the mean change in the BPI average pain score from baseline to endpoint, there was no significant interaction by age $(P=0.523)$, sex $(P=0.929)$, race $(P=0.382)$, diagnosis of MDD $(P=0.496)$ and secondary diagnosis of anxiety $(P=0.152)$. Among patients with a diagnosis of MDD, the mean change (SE) from baseline for duloxetine-treated patients was $-1.34(0.42)$ and in placebo-treated patients was -0.48 (0.46). For patients who did not have a diagnosis of MDD, 
Table 2 Least-squares (LS) mean change from baseline to endpoint or at endpoint: efficacy measures

Duloxetine $\quad$ Placebo
$60 / 120 \mathrm{mg}$ QD

\begin{tabular}{llllll}
\hline $\mathbf{N}$ & $\begin{array}{l}\text { LS mean } \\
\text { change }(\mathrm{SE})\end{array}$ & $\mathbf{N}$ & $\begin{array}{l}\text { LS mean } \\
\text { change }(\mathrm{SE})\end{array}$ & $\begin{array}{l}\text { Duloxetine-placebo } \\
\text { difference }(95 \% \mathrm{Cl})\end{array}$ & $\begin{array}{l}\text { P Value } \\
\end{array}$
\end{tabular}

\begin{tabular}{|c|c|c|c|c|c|c|c|}
\hline BPI average pain score & 158 & $-1.62(0.20)$ & 167 & $-1.13(0.19)$ & $-0.49(-0.99,0.01)$ & 0.053 & 0.015 \\
\hline BPI worst pain score & 158 & $-1.75(0.22)$ & 167 & $-1.25(0.21)$ & $-0.49(-1.04,0.05)$ & 0.077 & 0.019 \\
\hline BPI least pain score & 158 & $-1.22(0.19)$ & 167 & $-0.73(0.18)$ & $-0.49(-0.97,-0.01)$ & 0.046 & 0.114 \\
\hline $\mathrm{BPI}$ pain right now score & 158 & $-1.68(0.21)$ & 167 & $-1.16(0.20)$ & $-0.52(-1.05,0.01)$ & 0.054 & 0.261 \\
\hline $\mathrm{BPI}$ average interference & 158 & $-1.69(0.20)$ & 167 & $-1.03(0.19)$ & $-0.66(-1.16,-0.17)$ & 0.009 & 0.139 \\
\hline Mean tender point threshold & 148 & $0.40(0.09)$ & 159 & $0.18(0.08)$ & $0.22(-0.00,0.44)$ & 0.055 & 0.002 \\
\hline Count of low threshold & 148 & $-1.50(0.30)$ & 159 & $-0.92(0.28)$ & $-0.58(-1.32,0.16)$ & 0.121 & $<0.001$ \\
\hline FIQ total score & 153 & $-7.96(1.35)$ & 163 & $-5.81(1.29)$ & $-2.15(-5.57,1.28)$ & 0.218 & 0.088 \\
\hline FIQ fatigue score & 154 & $-0.94(0.23)$ & 163 & $-0.97(0.22)$ & $0.03(-0.54,0.60)$ & 0.919 & $0.74 I$ \\
\hline FIQ rested score & 154 & $-1.09(0.23)$ & 163 & $-1.06(0.22)$ & $-0.03(-0.61,0.54)$ & 0.916 & 0.779 \\
\hline FIQ physical impairment score & 154 & $-0.02(0.18)$ & 163 & $-0.06(0.17)$ & $0.03(-0.43,0.49)$ & 0.892 & 0.106 \\
\hline FIQ pain score & 154 & $-1.69(0.22)$ & 163 & $-1.06(0.22)$ & $-0.63(-1.20,-0.06)$ & 0.030 & 0.054 \\
\hline CGI-S & 156 & $-0.57(0.09)$ & 164 & $-0.28(0.08)$ & $-0.29(-0.51,-0.07)$ & 0.011 & 0.003 \\
\hline MFI general fatigue & 152 & $-0.33(0.30)$ & 162 & $-0.37(0.29)$ & $0.03(-0.73,0.80)$ & 0.930 & 0.090 \\
\hline MFI physical fatigue & 152 & $-0.48(0.30)$ & 162 & $-0.29(0.29)$ & $-0.19(-0.97,0.58)$ & 0.619 & 0.281 \\
\hline MFI mental fatigue & 153 & $-0.99(0.33)$ & 161 & $-0.03(0.31)$ & $-0.96(-1.79,-0.13)$ & 0.023 & 0.417 \\
\hline MFI reduced motivation & 152 & $-0.27(0.32)$ & 162 & $-0.07(0.3 \mathrm{I})$ & $-0.20(-1.01,0.61)$ & 0.629 & 0.213 \\
\hline MFI reduced activity & $|5|$ & $-0.19(0.34)$ & 162 & $0.03(0.33)$ & $-0.21(-1.09,0.66)$ & 0.632 & 0.158 \\
\hline HAMD $_{17}$ total score & 143 & $-2.04(0.40)$ & 158 & $-1.70(0.37)$ & $-0.34(-1.35,0.66)$ & 0.503 & 0.639 \\
\hline BDI-II total score & 154 & $-3.42(0.63)$ & 164 & $-1.45(0.61)$ & $-1.97(-3.58,-0.36)$ & 0.017 & 0.591 \\
\hline PGI-I & 158 & $3.43(0.13)$ & 165 & $3.72(0.12)$ & $-0.30(-0.62,0.03)$ & 0.073 & 0.004 \\
\hline AUC of pain relief ${ }^{b}$ & 162 & 270.07 (25.19) & 168 & $185.25(24.42)$ & $84.82(20.8 \mathrm{I}, \mathrm{I} 48.8)$ & 0.010 & 0.049 \\
\hline
\end{tabular}

Notes: ${ }^{\mathrm{F}}$ For PGI-I, endpoint was analyzed, and the baseline used as a covariate was the PGI-S; ${ }^{\mathrm{A}} \mathrm{AUC}$ of pain relief is a summary measure of change in the BPI average pain score over time, but is not a change from baseline to endpoint.

Abbreviations: QD, once daily; N, number of patients who had a baseline score (PGI-S for analysis of the PGI-I) and at least one nonmissing postbaseline score for that particular variable; SE, standard error; BPI, Brief Pain Inventory; FIQ, Fibromyalgia Impact Questionnaire; CGI-S, Clinical Global Impressions of Severity; MFI, Multidimensional Fatigue Inventory; HAMD ${ }_{17}$, I7-item Hamilton Depression Rating Scale; BDI-II, Beck Depression Inventory-II; PGI-I, Patient's Global Impressions of Improvement; AUC, area under the curve; SE, standard error; PGI-S, Patient's Global Impressions of Severity.

the mean change (SE) from baseline for duloxetine-treated patients was $-1.60(0.23)$ and in placebo-treated patients was $-1.20(0.22)$. There was a significant therapyby-previous antidepressant use interaction $(\mathrm{P}=0.029)$. Among patients with previous antidepressant use, the mean change (SE) in the BPI average pain score for duloxetinetreated patients was $-1.85(0.29)$ and in placebo-treated patients was $-0.65(0.27)$. For patients without previous antidepressant use, the mean change (SE) for duloxetinetreated patients was $-1.56(0.29)$ and in placebo-treated patients was $-1.51(0.27)$.

\section{Health outcomes and quality of life}

Duloxetine-treated patients had significantly greater improvements, compared with placebo-treated patients in the SF-36 mental component summary score (mean change: duloxetine 3.37 , placebo $0.79, P=0.026$ ), and SF-36 mental health score (mean change: duloxetine 6.63, placebo 1.19 , $P=0.005$ ) (Table 3).

\section{Safety}

For the 117 duloxetine-treated patients who continued past Week 13, the number of patients who stayed on duloxetine 
Table 3 Least-squares (LS) mean change from baseline to endpoint: health outcome and quality-of-life measures

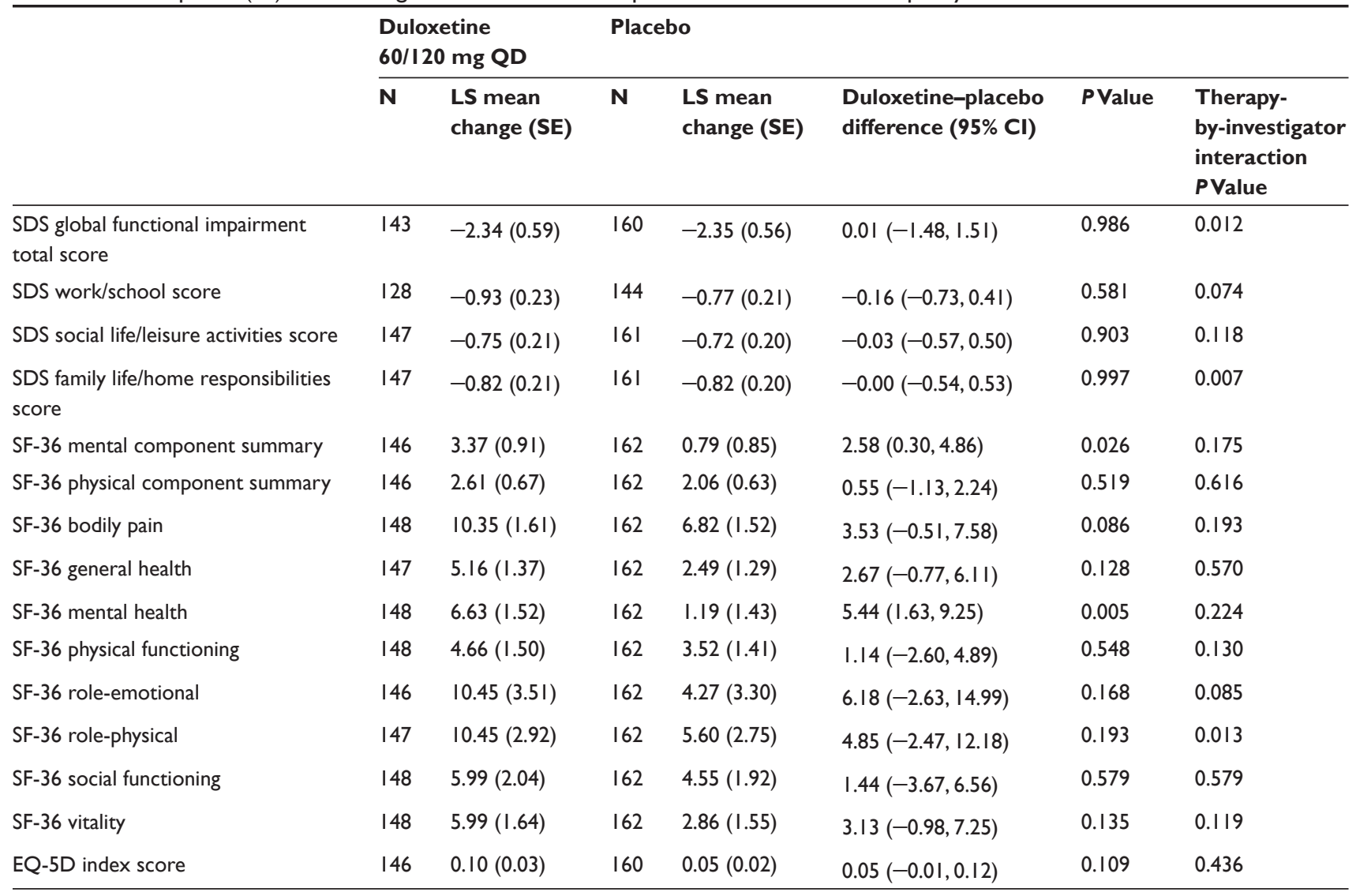

Abbreviations: QD, once daily; N, number of patients who had a baseline score and at least one nonmissing postbaseline score for that particular variable; SE, standard error; SDS, Sheehan Disability Scale; SF-36, 36-item Short-Form Health Survey; EQ-5D, EuroQol Questionnaire-5 dimensions.

$60 \mathrm{mg}$ QD were 19 (16.2\%) and those who escalated were $98(83.8 \%)$. The mean (SD) average prescribed daily dose between Weeks 13 and 27 for patients who escalated $(\mathrm{N}=97)$ was 113.4 (12.6) $\mathrm{mg}$.

Of the 330 randomly assigned patients, $145 / 162$ (89.5\%) duloxetine- and 137/168 (81.5\%) placebo-treated patients reported at least 1 TEAE $(P=0.043)$. TEAEs that occurred in $\geq 5 \%$ of duloxetine- and twice the rate of placebo-treated patients are shown in Table 4. There were no TEAEs that occurred at a significantly higher rate in the placebo- compared with the duloxetine-treated group. No deaths occurred during the study. No significant difference between treatment groups was observed in the percentage of patients with at least 1 serious adverse event (SAE). During the study, 4 patients (2.5\%) in the duloxetine-treated group experienced the SAEs of arthralgia, gait disturbance, pseudomonal lung infection, muscular weakness, paraesthesia, and pseudoneurologic symptom (1 [0.6\%] for each event), and 4 (2.4\%) patients in the placebo-treated group experienced the SAEs of arthralgia, abdominal pain upper, cystocele, and noncardiac chest pain (1 [0.6\%] for each event). A total of 49 (14.8\%) patients discontinued during the therapy phase due to an adverse event, with no significant difference between the duloxetine- and placebotreated groups (duloxetine, 30 [18.5\%]; placebo, 19 [11.3\%]; $P=0.088)$. The most common $(\geq 1 \%)$ adverse events reported as reasons for discontinuation in the duloxetinetreated group were nausea (3 $[1.9 \%])$, dizziness, diarrhea, lethargy, somnolence, and vomiting (2 [1.2\%] for each event), and in the placebo-treated group, dizziness and irritability (2 [1.2\%] for each event).

There were significant differences between the duloxetineand the placebo-treated groups for mean change in alkaline phosphatase (mean change [SD] units/liter: duloxetine $=2.38$ [12.02], placebo $=-2.45$ [9.94]; $P<0.001$ ), alanine transaminase/serum glutamate pyruvate transaminase (mean change [SD], units/liter: duloxetine, 6.97 [50.29]; placebo, -0.93 [5.70]; $P=0.038$ ), total bilirubin (mean change [SD], micromole/liter: duloxetine, -0.35 [2.97]; placebo, 0.27 [2.54]; $P=0.020$ ), cholesterol (mean change [SD], micromole/liter: duloxetine -0.04 [0.71]; placebo, -0.23 [0.75]; $P=0.016$ ), and uric acid (mean change [SD], 
Table 4 Treatment-emergent adverse events that occurred in $\geq 5 \%$ of duloxetine patients and at least twice the rate in placebo patients

\begin{tabular}{llll}
\hline & $\begin{array}{l}\text { Duloxetine } \\
\mathbf{N}=162, \mathbf{n}(\%)\end{array}$ & $\begin{array}{l}\text { Placebo } \\
\mathbf{N}=168, \mathbf{n}(\%)\end{array}$ & P Value* \\
\hline Nausea & $44(27.2)$ & $16(9.5)$ & $<0.001$ \\
Headache & $35(21.6)$ & $18(10.7)$ & 0.010 \\
Dry mouth & $32(19.8)$ & $9(5.4)$ & $<0.001$ \\
Diarrhoea & $26(16.0)$ & $11(6.5)$ & 0.008 \\
Constipation & $26(16.0)$ & $9(5.4)$ & 0.002 \\
Hyperhidrosis & $17(10.5)$ & $4(2.4)$ & 0.003 \\
Arthralgia & $10(6.2)$ & $5(3.0)$ & 0.193 \\
Somnolence & $12(7.4)$ & $2(1.2)$ & 0.006 \\
Dyspepsia & $9(5.6)$ & $4(2.4)$ & 0.164 \\
Sleep disorder & $9(5.6)$ & $4(2.4)$ & 0.164 \\
\hline
\end{tabular}

Note: *Fisher's exact $P$ value.

Abbreviation: $\mathrm{N}$, number of randomly assigned patients.

micromole/liter: duloxetine, -19.51 [43.50]; placebo, 3.91 [42.92]; $P<0.001)$.

Significant treatment group differences were observed for mean change in sitting pulse rate (mean change [SE], beats/minute: duloxetine, 1.08 [0.87]; placebo, -1.64 [0.84]; $P=0.016$ ) and diastolic BP (mean change [SE], mmHg: duloxetine, 1.68 [0.85]; placebo, -1.46 [0.82]; $P=0.004)$. Three patients in each group experienced sustained elevation of BP (defined as systolic BP $\geq 140$ and $\geq 10 \mathrm{mmHg}$ increase for at least 3 consecutive visits or diastolic $\mathrm{BP} \geq 90$ and $\geq 10 \mathrm{mmHg}$ increase for at least 3 consecutive visits), and the between-group difference was not significant.

No significant differences were observed between treatment groups in mean change for corrected QT intervals using either Fridericia's (QTcF), Bazett's (QTcB), or the regression correction, and in the QRS interval. Significant differences were observed between treatment groups in mean change in the PR interval (millisecond) from baseline to endpoint (mean change [SE]: duloxetine, -4.51 [1.20]; placebo, 1.97 [1.14]; $P<0.001$ ), QT interval (mean change [SE]: duloxetine, -4.27 [1.98]; placebo, 3.79 [1.87]; $P=0.002$ ), RR interval (mean change [SE]: duloxetine, -36.00 [10.73]; placebo, 14.51 [10.15]; $P<0.001$ ), and heart rate (mean change [SE] beats/minute: duloxetine, 2.87 [0.83]; placebo, -0.99 [0.79]; $P<0.001)$.

\section{Discussion}

In this phase-III, parallel, double-blind, 27-week, placebocontrolled trial, no statistically significant differences were observed between the treatment groups for the co-primary efficacy measures (mean change in BPI average pain score from baseline to endpoint and mean PGI-I score at endpoint). For these, and several other variables, there was a statistically significant treatment-by-investigator interaction (shown in Tables 2 and 3); therefore, the overall effect must be interpreted with caution. The nature of the interaction was investigated, and it could not be clearly explained by country-specific or geographic region (United States vs Europe) effects. The duloxetine group improved significantly more than the placebo group at all visits through Week 8 , as well as at Week 18, for the BPI average pain score and at all visits through Week 8 , as well as Weeks 18, 23, and 27 for the PGI-I.

The magnitude of the treatment benefit reported in the current study for the mean change in the BPI average pain score from baseline to endpoint and the PGI-I score at endpoint are not consistent with those reported in the previous clinical trials evaluating duloxetine in the treatment of fibromyalgia. ${ }^{25,26}$ Most previous pain studies were 12 weeks or less in duration. ${ }^{23-26}$ In the previous phase-II, 12-week, randomized, placebo-controlled trial of duloxetine in which male and female patients with ACRdefined primary fibromyalgia were randomized to duloxetine $60 \mathrm{mg}$ BID or placebo, ${ }^{25}$ duloxetine was superior to placebo on the BPI average pain score and PGI-I score. The previous phase-III study of female patients randomized to duloxetine $60 \mathrm{mg}$ QD, $60 \mathrm{mg}$ BID, or placebo ${ }^{26}$ also showed superiority of duloxetine compared with placebo on the BPI average pain score and the PGI-I score. The treatment effects for duloxetine compared with placebo for the co-primary efficacy variables of the BPI average pain score and the PGIImprovement in this study were smaller than were observed in three other placebo-controlled studies of duloxetine. Also, the treatment effects for almost all secondary measures were smaller than in the previous studies. Rater training and other aspects of study conduct were similar for all the studies. One difference between this and previous studies is the statistically significant treatment-by-investigator interactions for the mean change in both co-primary variables and for many secondary measures in this study. Eighteen of the 36 investigators had small numbers of patients with data and were pooled into one investigator for analysis, for which the results were numerically in favor of duloxetine compared with placebo for both the BPI average pain score and the PGI-Improvement. However, for the remaining 18 investigators, the results were numerically in favor of placebo compared with duloxetine for 10 investigators for 
the BPI and for seven investigators for the PGI-Improvement. This was the first study to include patients in the United States and also elsewhere (Germany, Spain, Sweden, and the United Kingdom). The interaction did not appear to be attributable to country or region (United States vs Europe). In spite of the treatment reversals for a large number of investigators, which dilutes the overall treatment effect, there were large benefits for duloxetine compared with placebo for some investigators, resulting in an overall numerical advantage for duloxetine for both the BPI average pain score and the PGI-Improvement, indicating the supportive nature of this study for a treatment benefit for duloxetine in the treatment of patients with fibromyalgia.

Compared with placebo-treated patients, patients treated with duloxetine had significantly greater AUC of pain relief and experienced greater improvements in the BPI least pain score and average interference score. In addition, duloxetinetreated patients experienced significantly greater improvements, compared with placebo, in the FIQ pain item, MFI mental fatigue dimension, CGI-S, and BDI-II total scores. Approximately $22 \%$ of all patients had MDD at baseline, which is consistent with the prevalence of depression concurrent with fibromyalgia $(22 \%-45 \%))^{4,40-43}$ No treatmentby-MDD interaction was observed for mean change in the BPI average pain score. In the present study there was no significant therapy-by-sex interaction, suggesting that the effect of duloxetine compared with placebo was similar in males and females. A previous clinical trial evaluating duloxetine in the treatment of fibromyalgia demonstrated a significant effect on reduction of pain in women but not in men, ${ }^{25}$ although this discrepancy might have been be due to the small number of men enrolled in the study.

Generalizability of these results is limited by the fact that patients in this study were carefully selected to exclude psychiatric and medical co-morbidities, and could be less severely ill than fibromyalgia patients in the general population.

In summary, in patients with fibromyalgia, with or without MDD, duloxetine 60/120 mg/day improved average pain severity and self-reported global improvement through week 8 (and some subsequent visits), but not at endpoint, relative to placebo. Consistent with earlier trials of duloxetine in the treatment of fibromyalgia, ${ }^{25,26}$ in this study, duloxetine was safely administered, and relatively well tolerated (considering the length of the trial) by most patients. Although duloxetine failed to demonstrate significant improvement over placebo on the co-primary outcome measures, in this supportive study, duloxetine demonstrated significant improvement compared with placebo on a number of secondary measures that are important in assessing treatment efficacy in patients with fibromyalgia.

\section{Acknowledgments}

This work was sponsored by Eli Lilly and Company and Boehringer Ingelheim GmbH. Trial Investigators were: Dr Gabriel Herrero-Beaumont, Fundación Jiménez Díaz; Dr Arturo Rodriguez De La Serna, Hospital Santa Creu I Sant Pau; Dr Cayetano Alegre De Miquel, Ciutat Sanitaria De La Vall De Hebron; Dr Juan Roberto Minguélez Sánchez, Hospital De Mostoles; Dr Javier Rivera Redondo, Instituto De Rehabilitación-Hospital Gregorio Marañón; Dr Roland Woerz (Office); Dr Wolfgang Bolten, Rheumaklinikum Wiesbaden-Zentrum Für Schmerztherapie; Dr Wolfgang Brueckle, Rheumaklinik Bad Nenndorg; Dr Gerhard Mueller-Schwefe (Office); Dr Wolfgang Molt (Practice); Dr Michael Ribbat (Office); Dr Walter Albrecht (Practice), Dr Michael Spaeth (Practice), Dr Olof Zachrisson, Gottfriesmottagningen; Dr Britt-Marie Öberg, Danderyds Sjukhus Rehabiliteringsmedicinska kliniken; Dr Jan Sorensen, Universitetssjukhuset Linkoping Smärt-och rehabcenter Smärtoch Rehabcenter; Dr Brian Hazleman, Addenbrookes Hospital; Dr Anthony D Woolf, Royal Cornwall Hospital; Dr Bruce Kirkham, Guys and St. Thomas Hospital Rheumatology Department; Dr Ashok Bhalla, Royal National Hospital For Rheumatic Diseases; Dr James R. Bellor, Columbia Medical Practice; Dr Laurence Bradley, University of Alabama at Birmingham; Dr Nicholas A. Bertini, Clinical Research Consultants, Inc.; Dr Mildred V. Farmer, Meridien Research; Dr H. S. Eugene Fung, Arthritis and Osteoporosis Clinic Of Central Texas; Dr Ronica Kluge, Clinical Physiology Associates, Clinical Study Center; Dr Andrew F. Leuchter, Neuropsychiatric Institute; Dr Lori Wynstock, Southern California Clinical Lab; Dr C Brendan Montano, Connecticut Clinical Trials Llc; Dr Paul K. Pickrell, Metaclin Research Inc.; Dr Steven Elliot, Medisphere Medical Research Center Llc; Dr Oscar Soto (Private); Dr Harris H. Mcilwain, Tampa Medical Group, P. A; Dr Craig M. Mccarthy, Pivotal Research Centers; Dr Siavash Nael, Neuropsychiatric Center; Dr Timothy R. Smith, Mercy Health Research.

\section{Disclosures}

Drs. Chappell, Detke, and D'Souza are employees and stockholders of Eli Lilly and Company. Dr Wiltse is a former employee of Eli Lilly and Company. Dr Spaeth is a consultant to Allergan, Eli Lilly, Jazz, and Pierre Fabre 
Medicament, and is on the speaker bureaus of Eli Lilly and Pierre Fabre Medicament. Dr Bradley is a consultant for Eli Lilly, Pfizer, and Forest; has received grant/research support from the National Institutes of Health, the Agency for Healthcare Research and Quality, Eli Lilly, Pfizer, and the American Fibromyalgia Syndrome Association; has received honoraria from Eli Lilly, Pfizer, Forest, and the Society for Women's Health Research; is a member of the speaker/advisory board for Pfizer; and has received royalties from UpToDate Rheumatology.

\section{References}

1. Wolfe F, Smythe HA, Yunus MB, et al. The American College of Rheumatology 1990 criteria for the classification of fibromyalgia. Report of the Multicenter Criteria Committee. Arthritis Rheum. 1990;33:160-172.

2. Hudson JI, Pope HG Jr. The relationship between fibromyalgia and major depressive disorder. Rheum Dis Clin N Am. 1996;22:285-303.

3. Blackburn-Munro G, Blackburn-Munro RE. Chronic pain, chronic stress and depression: coincidence or consequence? J Neuroendocrinol. 2001;13:1009-1023.

4. Wolfe F, Ross K, Anderson J, et al. The prevalence and characteristics of fibromyalgia in the general population. Arthritis Rheum. 1995;38:19-28.

5. Neumann L, Buskila D. Epidemiology of fibromyalgia. Curr Pain Headache Rep. 2003;7:362-368.

6. Basbaum AI, Fields HL. Endogenous pain control systems: brainstem spinal pathways and endorphin circuitry. Annu Rev Neurosci. 1984;7:309-338.

7. Clark FM, Proudfit HK. The projections of noradrenergic neurons in the A5 catecholamine cell group to the spinal cord in the rat: anatomical evidence that A5 neurons modulate nociception. Brain Res. 1993;616:200-210.

8. Coderre TJ, Katz J. Peripheral and central hyperexcitability: differential signs and symptoms in persistent pain. Behav Brain Sci. 1997;20:404-419; discussion 435-513.

9. Russell IJ, Michalek JE, Vipraio GA, et al. Platelet 3H-imipramine uptake receptor density and serum serotonin levels in patients with fibromyalgia/fibrositis syndrome. J Rheumatol. 1992;19:104-109.

10. Russell IJ, Vaeroy H, Javors M, et al. Cerebrospinal fluid biogenic amine metabolites in fibromyalgia/fibrositis syndrome and rheumatoid arthritis. Arthritis Rheum. 1992;35:550-556.

11. Yunus MB, Dailey JW, Aldag JC, et al. Plasma tryptophan and other amino acids in primary fibromyalgia: a controlled study. J Rheumatol. 1992;19:90-94.

12. Legangneux E, Mora JJ, Spreux-Varoquaux O, et al. Cerebrospinal fluid biogenic amine metabolites, plasma-rich platelet serotonin and $\left[{ }^{3} \mathrm{H}\right]$ imipramine reuptake in the primary fibromyalgia syndrome. Rheumatology (Oxford). 2001;40:290-296.

13. Wolfe F, Zhao S, Lane N. Preference for nonsteroidal antiinflammatory drugs over acetaminophen by rheumatic disease patients: a survey of 1,799 patients with osteoarthritis, rheumatoid arthritis, and fibromyalgia. Arthritis Rheum. 2000;43:378-385.

14. Yunus MB, Masi AT, Aldag JC. Short term effects of ibuprofen in primary fibromyalgia syndrome: a double blind, placebo controlled trial. $J$ Rheumatol. 1989;16:527-32. Erratum in: J Rheumatol. 1989;16:855.

15. Bennett RM, Gatter RA, Campbell SM, et al. A comparison of cyclobenzaprine and placebo in the management of fibrositis. A double-blind controlled study. Arthritis Rheum. 1988;31:1535-1542.

16. Goldenberg DL, Felson DT, Dinerman H. A randomized, controlled trial of amitriptyline and naproxen in the treatment of patients with fibromyalgia. Arthritis Rheum. 1986;29:1371-1377.
17. Wolfe F, Cathey MA, Hawley DJ. A double-blind placebo controlled trial of fluoxetine in fibromyalgia. Scand J Rheumatol. 1994; 23:255-259.

18. Gendreau RM, Thorn MD, Gendreau JF, et al. Efficacy of milnacipran in patients with fibromyalgia. J Rheumatol. 2005;32:1975-1985.

19. Seltzer Z, Dubner R, Shir Y. A novel behavioral model of neuropathic pain disorders produced in rats by partial sciatic nerve injury. Pain. 1990;43:205-218.

20. Kim SH, Chung JM. An experimental model for peripheral neuropathy produced by segmental spinal nerve ligation in the rat. Pain. 1992;50:355-363.

21. Bennett RM. Emerging concepts in the neurobiology of chronic pain: evidence of abnormal sensory processing in fibromyalgia. Mayo Clin Proc. 1999; 74:385-389.

22. Goldstein DJ, Lu Y, Detke MJ, et al. Effects of duloxetine on painful physical symptoms associated with depression. Psychosomatics. 2004;45:17-28.

23. Goldstein DJ, Lu Y, Detke MJ, et al. Duloxetine vs placebo in patients with painful diabetic neuropathy. Pain. 2005;116:109-118.

24. Wernicke JF, Pritchett YL, D'Souza DN, et al. A randomized controlled trial of duloxetine in diabetic peripheral neuropathic pain. Neurology. 2006;67:1411-1420

25. Arnold LM, Lu Y, Crofford LJ, et al. A double-blind, multicenter trial comparing duloxetine to placebo in the treatment of fibromyalgia patients with or without major depressive disorder. Arthritis Rheum. 2004;50:2974-2984

26. Arnold LM, Rosen A, Pritchett YL, et al. A randomized, double-blind, placebo-controlled trial of duloxetine in the treatment of women with fibromyalgia with or without major depressive disorder. Pain. 2005;119:5-15.

27. Cleeland CS, Ryan KM. Pain assessment: global use of the Brief Pain Inventory. Ann Acad Med Singap. 1994;23:129-138.

28. Guy W. ECDEU Assessment Manual for Psychopharmacology, Revised. Rockville (MD): US Dept of Health, Education, and Welfare, Public Health Service, Alcohol, Drug Abuse, and Mental Health Administration, National Institute of Mental Health, Psychopharmacology Research Branch, Division of Extramural Research Programs; 1976.

29. Burckhardt CS, Clark SR, Bennett RM. The fibromyalgia impact questionnaire: development and validation. J Rheumatol. 1991;18:728-733.

30. Fischer AA. Pressure threshold meter: its use for quantification of tender spots. Arch Phys Med Rehabil. 1986;67:836-838.

31. Smets EM, Garssen B, Bonke B, et al. The Multidimensional Fatigue Inventory (MFI) psychometric qualities of an instrument to assess fatigue. J Psychosom Res. 1995;39:315-325.

32. Hamilton M. A rating scale for depression. J Neurol Neurosurg Psychiatry. 1960;23:56-62.

33. Hamilton M. Development of a rating scale for primary depressive illness. Br J Soc Clin Psychol. 1967;6:278-296.

34. Beck AT, Epstein N, Brown G, et al. An inventory for measuring clinical anxiety: psychometric properties. J Consult Clin Psychol. 1988;56:893-897.

35. Sheehan D, Harnett-Sheehan K, Raj B. The measurement of disability. Int Clin Psychopharmacol. 1996;11(3 Suppl):89-95.

36. Ware JE, Snow KK, Kosinski M, et al. SF-36 health survey: manual and interpretation guide. Boston, MA: The Health Institute, New England Medical Center; 1993.

37. Kind P. The EuroQoL instrument: an index of health-related quality of life. In: Spilker B, editor. Quality of Life and Pharmacoeconomics in Clinical Trials. 2nd ed. Philadelphia (PA): Lippincott Williams and Wilkins; 1996. pp. 191-201.

38. Mallinckrodt $\mathrm{CH}$, Sanger TM, Dube' S, et al. Assessing and interpreting treatment effects in longitudinal clinical trials with missing data. Biol Psychiatry. 2003;53:743-760.

39. International Conference on Harmonization of Technical Requirements for Registration of Pharmaceuticals for Human Use. Medical Dictionary for Regulatory Activities Terminology (MedDRA). 2007. Accessed June 8,2007 . Available from: http://meddramsso.com. 
40. Celiker R, Borman P, Oktem F, et al. Psychological disturbance in fibromyalgia: relation to pain severity. Clin Rheumatol. 1997; 16:179-184.

41. Anderberg UM, Forsgren T, Ekselius L, et al. Personality traits on the basis of the Temperament and Character Inventory in female fibromyalgia syndrome patients. Nord J Psychiatry. 1999;53:353-359.
42. Epstein SA, Kay G, Clauw D, et al. Psychiatric disorders in patients with fibromyalgia. A multicenter investigation. Psychosomatics. 1999;40:57-63.

43. White KP, Nielson WR, Harth M, et al. Chronic widespread musculoskeletal pain with or without fibromyalgia: psychological distress in a representative community adult sample. J Rheumatol. 2002;29:588-594. 